\title{
High Levels of Human Immunodeficiency Virus Type 1 in Blood and Semen of Seropositive Men in Sub-Saharan Africa
}

\author{
John R. Dyer,* Peter Kazembe, Pietro L. Vernazza, \\ Bruce L. Gilliam,* Martin Maida, Dick Zimba, \\ Irving F. Hoffman, Rachel A. Royce, Jody L. Schock, \\ Susan A. Fiscus, Myron S. Cohen, and Joseph J. Eron, Jr.
}

\begin{abstract}
Department of Medicine, Department of Epidemiology, and Department of Microbiology and Immunology, University of North Carolina, Chapel Hill, North Carolina; Department of Medicine, Kantonsspital, St. Gallen, Switzerland; Lilongwe Central Hospital, Lilongwe, Malawi
\end{abstract}

\begin{abstract}
High levels of human immunodeficiency virus type 1 (HIV-1) replication, as reflected in HIV-1 RNA concentrations in blood and semen, probably contribute to both rapid disease progression and enhanced sexual transmission. Semen and blood were collected from 49 Malawian and 61 US and Swiss (US/Swiss) HIV-1 - seropositive men with similar CD4 cell counts and no urethritis or exposure to antiretroviral drugs. Median seminal plasma and blood plasma HIV-1 RNA concentrations were $>3$-fold $(P=.034)$ and 5-fold $(P=.0003)$ higher, respectively, in the Malawian men. Similar differences were observed in subsets of the Malawian and US/Swiss study groups matched individually for CD4 cell count $(P=.035$ and $P<.002$, respectively). These observations may help explain the high rates of HIV-1 sexual transmission and accelerated HIV-1 disease progression in subSaharan Africa.
\end{abstract}

Most human immunodeficiency virus type 1 (HIV-1)-infected people live in regions of Africa and Asia, where heterosexual transmission predominates [1]. The continued rapid expansion of this heterosexual epidemic likely reflects interactions among many behavioral and biologic factors. Analogous to other sexually transmitted diseases (STDs), the HIV-1 inoculum in semen is probably a major determinant of transmission from men. In support of this hypothesis, plasma HIV-1 RNA concentrations, which often predict semen levels [2, 3], correlate with transmission between stable partners [4]. Mucosal STDs significantly increase seminal plasma viral RNA [5], helping to explain their association with HIV transmission.

HIV-1 disease may progress faster in sub-Saharan Africa than in North America and western Europe [6]. Single plasma

Received 4 August 1997; revised 6 January 1998.

Presented in part: 4th Conference on Retroviruses and Opportunistic Infections, Washington DC, 22-26 January 1997 (abstract 26).

Informed consent was obtained from all patients participating in this study. This research was conducted according to the human experimentation guidelines of the US Department of Health and Human Services. The protocol was approved by the University of North Carolina Committee on the Protection of Human Rights and The Malawi Health Sciences Research Committee.

Information contained herein does not necessarily reflect the views or policies of the US Agency for International Development.

Financial support: US Agency for International Development (USAID) as part of Family Health International's AIDS Control and Prevention (AIDSCAP) Project (623-02380A-00-4031-00); World Health Organization (award SD1/94/009); NIH (UO-31496 and DK-49381), NIH Office of AIDS Research; Pfizer; Swiss National Science Foundation (32-38818.93).

Reprints or correspondence: Dr. Joseph J. Eron, Jr., Dept. of Medicine, CB\# 7030, 547 Burnett-Womack Bldg., University of North Carolina, Chapel Hill, NC 27599-7030 (jeron@med.unc.edu).

* Present affiliations: Infectious Diseases Unit, Townsville General Hospital, Queensland, Australia (J.R.D.); The Henry M. Jackson Foundation, Rockville, Maryland (B.L.G.).

The Journal of Infectious Diseases $1998 ; 177: 1742-6$ (C) 1998 by The University of Chicago. All rights reserved. 0022-1899/98/7706-0045\$02.00
HIV-1 RNA measures independently predict prognosis in North American and European populations [7], but HIV-1 RNA concentrations in sub-Saharan Africans have not previously been reported. In this study, we examined the hypothesis that levels of HIV-1 in blood and semen in a Malawian population sampled in a cross-sectional fashion would exceed those in a comparable group of North American and European men, providing a biologic basis for the observed heterogeneity in rates of HIV-1 transmission and progression.

\section{Materials and Methods}

Study population. Forty-nine consecutive HIV-1-seropositive men attending dermatology clinics at Lilongwe Central Hospital (Malawian subjects) who served as a control group for a previously reported study [5] and $61 \mathrm{HIV}-1$-seropositive male volunteers from infectious diseases clinics at the University of North Carolina and the Kantonsspital, St. Gallen (US/Swiss subjects), were studied. The $61 \mathrm{US} / \mathrm{Sw}$ iss men were an antiretroviral naive subset of a larger cohort of men studied [3]. Clinic evidence of mucosal or ulcerative STDs led to exclusion from the study. In Malawian men, urethral infection with Neisseria gonorrhoeae, Trichomonas vaginalis, or Chlamydia trachomatis was excluded by Gram's stain, culture, and $C$. trachomatis ligase chain reaction (Uriprobe LCR; Abbott Diagnostics, Abbott Park, IL) of urethral swabs. Malawian men were confirmed as HIV-1-seropositive by two EIAs (HIV-1/HIV-2 EIA; Genetic Systems, Seattle; Murex HIV$1+2$, Murex Diagnostics, Dartford, UK), followed by Western blot (Organon Teknika, Durham, NC) for results that were equivocal. HIV-1 seropositivity was confirmed in all US/Swiss men by Western blot.

Specimen collection and laboratory procedures. Semen was processed as previously described [2]. US/Swiss samples were diluted in $2.5 \mathrm{~mL}$ of transport medium before processing. Blood plasma (BP) was separated and frozen at $-70^{\circ} \mathrm{C}$ within 1 day of semen collection. HIV-1 RNA concentrations in BP and cell free 
seminal plasma (SP) were quantified using nucleic acid sequencebased amplification assay (NASBA; Organon Teknika, Boxtel, The Netherlands) [2], with a sensitivity of 1000 RNA copies/mL.

$\mathrm{T}$ cell subsets were measured in blood collected from Malawian men at the same time as BP for HIV-1 RNA quantification. In the US/Swiss men, the CD4 cell count closest to the time of semen collection, within 1 month in each case, was used. HIV-1 subtype in Malawian men was determined by envelope V3 peptide immunoassay using antigens specific for clades A through F [8].

Statistics. Samples containing undetectable HIV-1 RNA were assigned a value half the assay detection limit (500 copies $/ \mathrm{mL}$ ). Arithmetic correction for dilution was made, except for undetectable RNA levels. The Mann-Whitney $U$ test was used for comparisons between groups and Wilcoxon rank sum test for paired comparisons. For paired analysis, individual Malawian and US/Swiss subjects were matched for CD4 cell counts within 10 cells $/ \mu \mathrm{L}$ of one another without knowledge of BP and SP HIV-1 RNA concentrations. Linear regression was used to identify independent predictors of BP and SP HIV-1 RNA.

\section{Results}

Characteristics of patient populations. Table 1 shows clinical and demographic characteristics of the subjects. In all but 5 cases, dermatologic disorders in Malawian men were minor, including superficial mycosis, seborrheic dermatitis, superficial bacterial infection, urticaria, pityriasis rosea, warts, molluscum contagiosum, eczema, psoriasis, and drug eruption. Five men had herpes zoster or primary varicella infection. No US/Swiss man described significant symptoms at the time of sample collection.

Comparisons of HIV-1 RNA concentrations in blood and semen. $\quad$ SP samples were collected from 49 Malawian and 61

Table 1. Clinical and demographic characteristics of 49 Malawian and 61 US and Swiss (US/Swiss) HIV-1-seropositive men.

\begin{tabular}{|c|c|c|c|}
\hline & Malawian & $\begin{array}{l}\text { US/Swiss } \\
\text { treatment-naive }\end{array}$ & $P^{*}$ \\
\hline Mean age (years) $\pm S D$ & $32 \pm 7.5$ & $35 \pm 8.0$ & .017 \\
\hline $\begin{array}{l}\text { Median CD4 cell count } \\
\quad(\text { cells } / \mu \mathrm{L}) \text {, interquartile range }\end{array}$ & $305(190-479)$ & $339(160-500)$ & .73 \\
\hline Asymptomatic (CDC stage A) & $36(73 \%)$ & $52(85 \%)$ & .15 \\
\hline $\begin{array}{l}\text { Symptomatic } \\
\text { (CDC stage B or C) }\end{array}$ & $13(27 \%)$ & $9(15 \%)$ & \\
\hline Active systemic infection & $8(16 \%)^{\dagger}$ & 0 & \\
\hline Mode of HIV-1 acquisition & ND & & \\
\hline Heterosexual & & $14(23 \%)$ & \\
\hline Homosexual & & $37(61 \%)$ & \\
\hline Intravenous drug users & & $10(16 \%)$ & \\
\hline
\end{tabular}

NOTE. In the Malawian group, 41 subjects had absolute CD4 cell counts available, and 42 had CD4 percentages available. In the US/Swiss group, all had CD4 cell counts done. ND, not determined.

* Continuous data were compared by Mann-Whitney $U$ test, and dichotomous variables were compared by Fisher's exact test.

${ }^{\dagger}$ Includes 4 subjects with dermatomal herpes zoster, 1 with disseminated varicella-zoster infection, 1 with secondary syphilis, 1 with active tuberculosis and possible drug-induced lupus, and 1 with possible tuberculosis (chronic cough, fever, night sweats, and weight loss.)
US/Swiss men and BP from 42 and 49 men, respectively. Significantly higher levels of HIV-1 RNA were observed in Malawian than in US/Swiss BP and SP (median, $15.5 \times 10^{4}$ copies/mL vs. $2.88 \times 10^{4}$ copies $/ \mathrm{mL}, P=.0003$; and $1.51 \times$ $10^{4}$ copies $/ \mathrm{mL}$ vs. $0.46 \times 10^{4}$ copies $/ \mathrm{mL}, P=.034$, respectively, figure 1A).

Paired analysis compared SP RNA concentrations in 33 pairs of Malawian and US/Swiss subjects individually matched for CD4 cell count (median CD4 cell counts, 299 cells $/ \mu \mathrm{L}$ and $300 / \mu \mathrm{L}$, respectively). BP HIV-1 RNA levels were available for 22 of these pairs. As shown in figure 1B, median Malawian SP HIV-1 RNA concentration was $1.70 \times 10^{4}$ copies $/ \mathrm{mL}$ compared with 500 copies/mL (below detectable) in US/Swiss men $(P=.035)$, with a median BP RNA of $15.5 \times 10^{4}$ copies $/ \mathrm{mL}$ versus $2.34 \times 10^{4}$ copies $/ \mathrm{mL}(P<.003)$.

Distributions of SP and BP HIV-1 RNA concentrations in the two study populations are illustrated in figures $1 \mathrm{C}$ and D. Fifty-seven percent (24/42) of Malawian men had blood HIV1 RNA levels $>125,000$ copies/mL compared with only $20 \%$ (10/49) of US/Swiss men ( $P=.0005$, figure 1D). The BP RNA level was below the assay detection limit of 1000 copies $/ \mathrm{mL}$ in $12 \%$ of US/Swiss compared with $10 \%$ of Malawian men; SP RNA was undetectable in $42 \%$ and $22 \%$, respectively. The differences between BP and SP log HIV-1 RNA values (equivalent to ratios between absolute BP and SP RNA levels) of individual subjects were similar in Malawian and US/Swiss groups (data not shown).

Malawian origin and CD4 cell count correlated significantly with the BP HIV-1 RNA concentration and independently predicted BP RNA level in multiple regression analysis (adjusted total $R^{2}=.35, P<.0001$ for absolute CD4 cell count, $P=$ .004 for country of origin). Although Malawian origin, CD4 cell count, BP RNA, and HIV-1 disease stage all individually correlated with SP HIV-1 RNA, the sole independent predictor following multiple linear regression analysis was BP RNA concentration (adjusted total $R^{2}=.24, P=.0012$ ).

Eight Malawian men had evidence of active systemic infections at the time of sampling (see table 1 footnote), while all US/Swiss subjects were clinically well. The actively infected men had marginally higher BP HIV-1 RNA levels than the remainder of the Malawian group (median, $3.24 \times 10^{5}$ copies/ $\mathrm{mL}[n=7]$ compared with $1.41 \times 10^{5}$ copies $\left./ \mathrm{mL}, P=.39\right)$ and similar SP RNA concentrations (median, $1.35 \times 10^{4}$ vs. $1.51 \times 10^{4}$ copies $\left./ \mathrm{mL}, P=.98\right)$. When these 8 men were excluded from analysis, both BP and SP HIV-1 RNA concentrations remained significantly higher in Malawian than US/ Swiss men $(P<.002$ and $P=.04$, respectively). The same observation held for paired analysis with matching for CD4 cell count, when the five pairs including an actively infected Malawian patient were excluded $(P=.008$ for comparison of BP HIV-1 RNA concentrations and $P=.006$ for SP RNA).

Of 42 Malawian men whose samples were submitted for subtype analysis, 40 had typeable virus. Thirty-seven had antibody directed only to peptide antigen specific for clade C, 1 had 

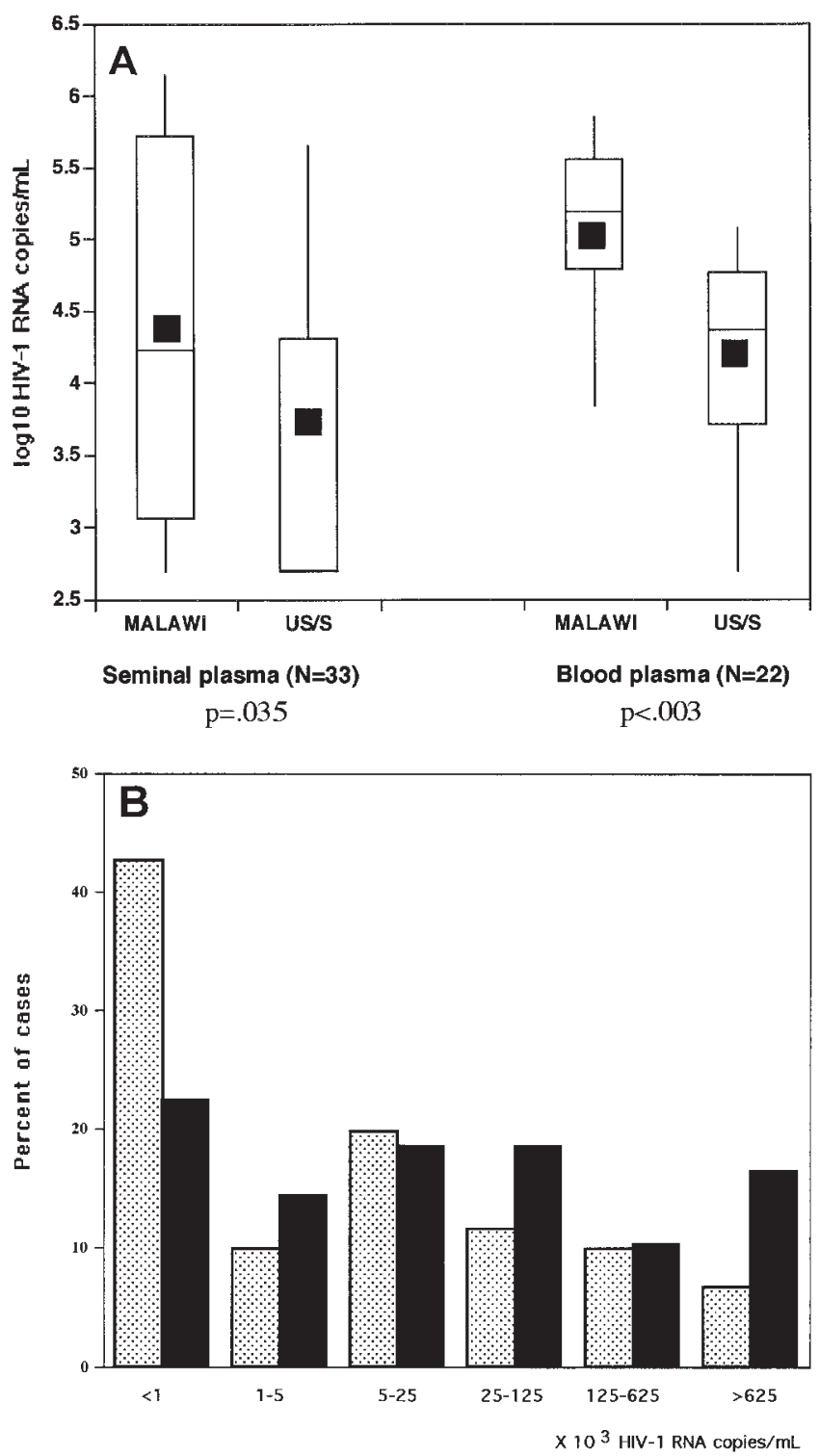

antibodies cross-reactive to clades A and C, 1 had antibodies to clades A, C, and F, and 1 had antibody to clade A only. All US/Swiss men had acquired HIV-1 infection in the United States or Switzerland and were considered likely to harbor clade B virus [1].

\section{Discussion}

We observed a 5- to 7-fold higher median BP viral RNA concentration in HIV-1-infected Malawian men than in a group of untreated US and Swiss men with similar CD4 cell counts. Recent studies indicate that blood levels of viral RNA are powerful predictors of progression to AIDS and death independent of CD4 cell count and other markers and that reductions in plasma HIV-1 RNA after antiviral drug therapy corre-
Figure 1. Comparison of blood (BP) and seminal plasma (SP) HIV-1 RNA concentrations (expressed as $\log _{10}$ values) in antiretroviral-naive Malawian and US/Swiss groups. Distribution of values is depicted in boxplots for subgroups matched individually for absolute CD4 cell count (A) and by histograms of $\log _{10}$ SP (C) and BP (D) HIV-1 RNA concentrations in US/Swiss (speckled bars) and Malawian (filled bars) subjects, plotted in multiples of 5 above detection limit of $1000 \mathrm{copies} / \mathrm{mL}$. In boxplots, horizontal lines represent median (25th and 75th percentiles), vertical lines extend to 10th and 90th percentiles, and small filled box is mean (calculated using 500 RNA copies/mL for values below assay detection limit).

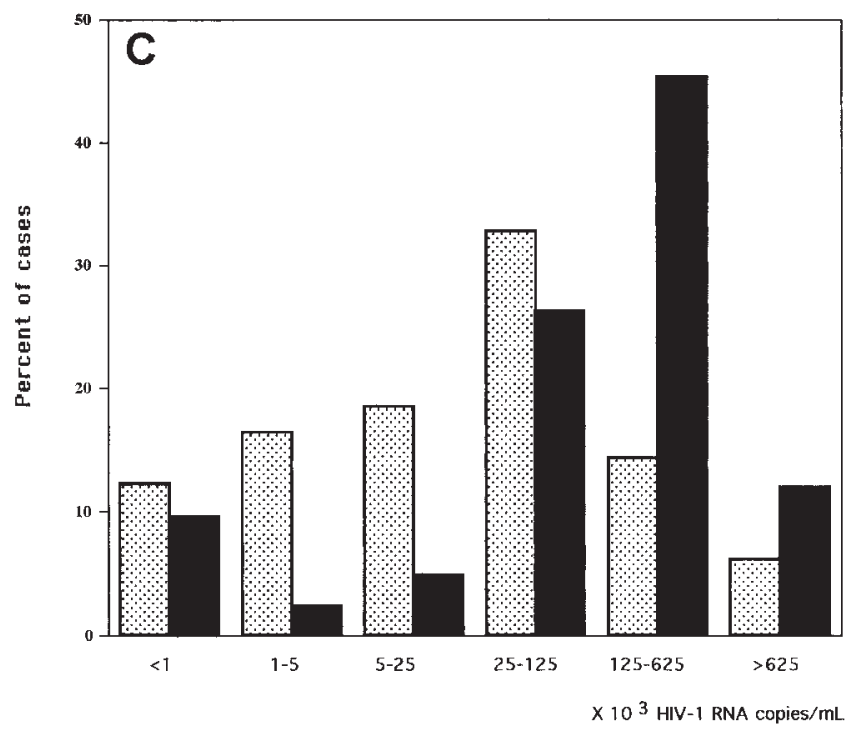

late with improved prognosis [7]. We found Malawian origin and residence to be a highly significant predictor of the BP HIV-1 RNA concentration, independent of CD4 cell count. High levels of HIV-1 replication could account for more rapid disease progression in sub-Saharan Africa than in the United States and Europe.

High levels of viral shedding in semen may partly explain the continuing rapid heterosexual spread of HIV-1 in sub-Saharan Africa [1]. We found 3- to 4-fold higher concentrations of SP HIV-1 RNA in Malawian men than in CD4 cell level-matched US/Swiss subjects receiving no antiretroviral therapy. In both groups, HIV-1 RNA levels in blood correlated with those in $\mathrm{SP}$, the BP concentration being the only independent predictor of seminal HIV-1 shedding. Blood-to-semen HIV-1 RNA ratios did not differ between the 2 groups, suggesting that similar 
factors were responsible for increased levels of virus in both body fluids in the Malawian men. Whether production of genital tract HIV-1 occurs in a separate biologic compartment, as suggested by previous studies [9], cannot be determined by the data presented here.

A number of host- or virus-related factors could explain the differences observed between BP and SP HIV-1 RNA levels of the 2 groups. Immune activation by infection or vaccination can reversibly elevate BP viral RNA concentrations [10]. Although 8 Malawian subjects had evidence of active systemic infection, they did not account for the elevated blood and semen RNA levels in the group as a whole. Nevertheless, common endemic infections such as tuberculosis and parasitic diseases could enhance viral replication while remaining clinically inapparent. High levels of serum immune activation markers have been described in HIV-1-infected Africans [11]. Furthermore, HIV-1-infected African immigrants to the United Kingdom progress at similar rates as seropositive Caucasians [12], suggesting strong environmental influences on the natural history of the disease.

Host genetic factors also influence HIV-1 pathogenesis and transmission. For example, heterozygosity for the $\Delta 32 \mathrm{CCR}-$ 5 gene polymorphism slows progression to AIDS and is associated with reduced levels of plasma viral RNA [13]. The occurrence of the polymorphic CCR-5 allele has not been described in populations without Caucasian ancestry. HLA-linked and other genetic immune polymorphisms may also contribute to various rates of virus replication and disease progression between different populations.

Various efficiencies of viral replication between divergent viral subtypes in lymphoid and genital tract cells could influence the natural history and transmission of HIV-1. Soto-Ramirez et al. [14] recently reported that HIV-1 subtype E infects and replicates in epithelial Langerhans' cells more efficiently in vitro than do clade B viruses. The fact that we did not observe disproportionately higher levels of HIV-1 in the genital tract compared with that in blood in African men argues against more efficient in vivo genital tract replication of specific viral subtypes.

There were a number of limitations inherent in our study design and methods. Unequal priming efficiencies of subtypes $\mathrm{B}$ and $\mathrm{C}$ with NASBA could produce artifactual differences in viral RNA concentrations, although NASBA appears to be equally sensitive for all $\mathrm{M}$ group HIV-1 subtypes except clades $A$ and $\mathrm{G}$ [15]. Given that the assay primers were designed and optimized to quantify clade B, differing amplification efficiencies should underestimate clade C HIV-1 RNA and bias against increased levels in the Malawian group. The relatively high assay detection limit resulted in censoring of information $<1000$ HIV-1 RNA copies/mL, and this result occurred more commonly in US/Swiss men. Finally, the retrospective and cross-sectional nature of the study and the necessity of selecting a comparison group of antiretroviral drug-naive US/Swiss men, who may have had a relatively stable disease course, caution against overinterpretation of our results.
The causes of the expanding heterosexual HIV-1 epidemics in sub-Saharan Africa and southern Asia are not clear. Behavioral factors may provide a partial explanation. However, distinct biologic aspects of the virus, the host, and the environment may be important determinants of regional patterns of HIV-1 spread and disease progression. The high HIV-1 RNA concentrations in blood and semen in this African population probably reflect interactions among genetic, immunologic, environmental, and viral influences. Modifiable environmental factors, especially acute and chronic bacterial and parasitic infections, may result in immune activation and increased systemic and genital tract HIV RNA concentrations. Dissecting the relative importance of these influences should enable development of new public health initiatives to control this epidemic.

\section{Acknowledgments}

We thank Jerry Russell and Peter Killick of John Snow Inc./ STAFH Project, Lilongwe, and Terrie Taylor of the Malaria Project, Blantyre, Malawi, for administrative and logistic support; John Schmitz and Michelle Fiordisi of the Department of Microbiology and Immunology, University of North Carolina Hospitals, and Elaine Doherty-Leach and Lance Nkana from the American Embassy Clinic in Malawi for technical and laboratory support; and Carol Porter of the Sheps Center, Chapel Hill, for assistance with data management. We would like to especially thank Gina Dallabetta of Family Health International's AIDS Control and Prevention Project and Lester Chitsulo of the Malawi AIDS Secretariat for their careful review of the manuscript.

\section{References}

1. Quinn TC. Global burden of the HIV pandemic. Lancet 1996;348: 99-106.

2. Dyer JR, Gilliam BL, Eron JJ, Grosso L, Cohen MS, Fiscus SA. Quantitation of human immunodeficiency virus type 1 RNA in cell free seminal plasma: comparison of NASBA with Amplicor reverse transcriptionPCR amplification and correlation with quantitative culture. J Virol Methods 1996;60:161-70.

3. Vernazza PL, Gilliam BL, Dyer JR, et al. Quantification of HIV in semen: correlation with antiviral treatment and immune status. AIDS 1997;11: 987-93.

4. Lee TH, Sakahara N, Fiebig E, Busch MP, O'Brien TR, Herman SA. Correlation of HIV-1 RNA levels in plasma and heterosexual transmission of HIV-1 from infected transfusion recipients. J Acquir Immune Defic Syndr Hum Retrovirol 1996; 12:427-8.

5. Cohen MS, Hoffman IF, Royce RA, et al. Reduction of concentration of HIV-1 in semen after treatment of urethritis: implications for prevention of sexual transmission of HIV-1. Lancet 1997;349:1868-73.

6. Anzala OA, Nagelkerke NJD, Bwayo JJ, et al. Rapid progression to disease in African sex workers with human immunodeficiency virus type 1 infection. J Infect Dis 1995; 171:686-9.

7. Saag MS. Use of HIV viral load in clinical practice: back to the future. Ann Intern Med 1997; 126:983-5.

8. Pau CP, Kai M, Holloman-Candal DL, et al. Antigenic variation and serotyping of HIV type-1 from four World Health Organization-sponsored HIV vaccine sites. AIDS Res Hum Retroviruses 1994; 10: $1369-77$. 
9. Zhu T, Wang N, Carr A, et al. Genetic characterization of human immunodeficiency virus type 1 in blood and genital secretions: evidence for viral compartmentalization and selection during sexual transmission. J Virol 1996; 70:3098-107.

10. Goletti D, Weissman D, Jackson RW, et al. Effect of Mycobacterium tuberculosis on HIV replication. Role of immune activation. J Immunol 1996; $157: 1271-8$.

11. Rizzardini G, Piconi S, Ruzzante S, et al. Immunological activation markers in the serum of African and European HIV-seropositive and seronegative individuals. AIDS 1996; 10:1535-42.
12. Del Amo J, Petruckevitch A, Phillips AN, et al. Spectrum of disease in Africans with AIDS in London. AIDS 1996; 10:1563-9.

13. Huang Y, Paxton WA, Wolinsky SM, et al. The role of a mutant CCR5 allele in HIV-1 transmission and disease progression. Nat Med 1996; $2: 1240-3$.

14. Soto-Ramirez LE, Renjifo B, McLane MF, et al. HIV-1 Langerhans' cell tropism associated with heterosexual transmission of HIV. Science 1996; $271: 1291-3$

15. Alaeus A, Lidman K, Sonnerborg A, Albert J. Subtype-specific problems with quantification of plasma HIV-1 RNA. AIDS 1997;11:859-65.

\title{
Proinflammatory Cytokine and Human Immunodeficiency Virus RNA Levels during Early Mycobacterium avium Complex Bacteremia in Advanced AIDS
}

\author{
David W. Haas, Michael M. Lederman, Lisa A. Clough, \\ Robert S. Wallis, David Chernoff, and Sheri L. Crampton
}

\author{
Department of Medicine, Microbiology, and Immunology, Division of \\ Infectious Diseases, Vanderbilt University School of Medicine, \\ Nashville, Tennessee; Department of Medicine, Division of Infectious \\ Diseases, Case Western Reserve University School of Medicine, \\ Cleveland, Ohio; Chiron Corp., Emeryville, California; \\ Abbott Laboratories, Abbott Park, Illinois
}

\begin{abstract}
The relationship between Mycobacterium avium complex (MAC) bacteremia and proinflammatory cytokine and human immunodeficiency virus type 1 (HIV-1) RNA levels in AIDS was investigated. During a prospective study, blood samples were drawn monthly for mycobacterial cultures. Sera were available at baseline and onset of MAC bacteremia from 20 cases and at corresponding times from 19 controls. Mean interleukin-6 (IL-6) levels were 154\% greater at the time of MAC bacteremia in cases than in controls. The IL-6 levels correlated with body temperature, serum tumor necrosis factor (TNF- $\alpha$ ) levels, and alkaline phosphatase levels ( $P \leqslant .004$ for each). Although TNF- $\alpha$ levels tended to rise more in MAC patients than in controls, the difference was not significant. However, among both cases and controls, serum TNF- $\alpha$ levels rose significantly from baseline to the time of last sample, irrespective of MAC infection $(P=.015)$. Bacteremia was not associated with increased serum HIV-1 RNA levels. Thus, early MAC bacteremia is associated with increases in serum IL-6 levels, while TNF- $\alpha$ levels rise over time during advanced AIDS.
\end{abstract}

Disseminated Mycobacterium avium complex (MAC) disease is common during advanced AIDS. Cellular expression of tumor necrosis factor- $\alpha$ (TNF- $\alpha)$ is induced by mycobacterial proteins in vitro [1] and during tuberculosis in human immunodeficiency virus (HIV)-infected persons [2]. In addition, immune activating events such as febrile illnesses and immunizations cause transient increases in plasma HIV RNA. Cytokines that promote HIV expression in vitro, such as TNF- $\alpha$ and interleukin-6 (IL-6), may enhance HIV replication [3]. Among

Received 28 February 1997; revised 18 December 1997.

This study was performed with the written approval of the Vanderbilt University Committee for the Protection of Human Subjects, following the guidelines of this institution.

Grant support: Abbott Laboratories.

Reprints or correspondence: Dr. David W. Haas, Dept. of Medicine, Microbiology, and Immunology, Vanderbilt University School of Medicine, 1211 21st Ave. S., Suite 539, Nashville, TN 37212 (david.w.haas@vanderbilt.edu).

The Journal of Infectious Diseases 1998; 177:1746-9

(C) 1998 by The University of Chicago. All rights reserved

0022-1899/98/7706-0046\$02.00
HIV-infected patients, active viral replication drives progressive CD4 cell depletion [4]. Current treatment strategies focus on minimizing HIV replication. The present study investigated whether early MAC bacteremia was associated with changes in serum IL-6, TNF- $\alpha$, and HIV RNA levels in patients with AIDS.

\section{Materials and Methods}

Patients. Patients previously enrolled in a prospective clinical trial of clarithromycin prophylaxis in AIDS were studied [5]. Patients with $<100 \mathrm{CD}^{+}$cells $/ \mathrm{mm}^{3}$ and baseline blood cultures negative for MAC were randomized to twice daily clarithromycin or placebo. Only patients from the placebo arm were included in the present analysis. Blood was drawn monthly for MAC surveillance cultures, and sera were sampled every 16 weeks. All patients with adequate stored sera and for whom matched controls could be identified were included. We identified 20 patients who developed MAC bacteremia (cases). A control was selected for each (except 1 patient who was a control for 2 cases). Cases and controls were 